\title{
The stability of intact parathyroid hormone in human blood during different sampling conditions and long- term storage in serum tubes
}

(1)

\section{Introduction}

- Measurement of circulating parathyroid hormone (PTH) is important in the evaluation of compensatory hyperparathyroidism caused by vitamin $D$ deficiency.

- The instability of PTH in plasma or serum ex vivo is, however, a matter of concern and pre-analytical conditions for PTH determination are important factors for the confidence of the assay (1)

- Only few published studies on the stability of PTH in human serum samples exist where a delay was present before processing and storage of samples (1-5). However, no information is available on the effect of long-term storage for up to five years in frozen serum samples on PTH.

- We therefore aimed to examine 1) the analytic stability of PTH in venous blood specimens in serum tubes processed at different time intervals after collection compared to immediately processed blood samples collected in K2EDTA tubes, and 2) whether storage of samples in serum tubes for five years at $-20^{\circ} \mathrm{C}$ had an effect on stability of PTH.

\section{Methods}

Venous blood samples were collected from 10 healthy Caucasian females (mean age, 38.8 years; SD, 10.6 years) into VacutainerTM tubes (Becton Dickinson Ltd, US) that contained either potassium EDTA (K2EDTA) or silica/gel for serum (SSTTM II Advance).

- For each participant, samples were collected in one K2EDTA tube and three serum tubes. The K2EDTA tube was prepared immediately and stored at $-20^{\circ} \mathrm{C}$. The serum tubes sampled were processed at three different time intervals after collection (30, 60 and 120 minutes). Subsequently, all samples were frozen at $-20^{\circ} \mathrm{C}$ for a minimum of 5 days before biochemical analyzes were performed.

To compare the storage condition and potential influence on PTH content over time, 100 serum samples obtained between September 2007 and February 2008, stored at $-20^{\circ} \mathrm{C}$, were analyzed in November 2012. These samples were compared to the PTH normal range reference $(8.3-68 \mathrm{pg} / \mathrm{mL})$ and to 100 matched samples collected and analyzed in K2EDTA tubes from March to April 2007.

Intact (1-84) PTH was analyzed in all samples using Elecsys PTH immunoassay technique (Roche Diagnostics, Mannheim, Germany) with a measurement range of $1.20-5000 \mathrm{pg} / \mathrm{mL}$. The intra- and inter-assay coefficients of variation were $<2.0 \%$ and $<3.4 \%$, respectively.

\section{Results}

- No significant difference was found between plasma samples in K2EDTA tubes processed immediately and serum tubes processed after 30,60 and 120 minutes of collection (see Table 1 and Fig. 1).

- Using plasma samples processed immediately after collection in the 10 healthy subjects as gold standard, the retrieval in serum samples processed 30 minutes after collection was $91.8 \%$. Serum processed 60 and 120 minutes after collection showed retrieval of $91.9 \%$ and $91.1 \%$, respectively, with no time trend $(p>0.84)$.

Table 1 shows retrieval in percentage of the absolute PTH values.

\begin{tabular}{lcccc}
\hline Analytic Preparation & $\begin{array}{c}\text { PTH }(\mathrm{pg} / \mathrm{mL}) \\
\text { Mean } \pm \text { SD }\end{array}$ & $\begin{array}{c}\text { Retrieval, } \% \\
\text { Mean }(95 \% \mathrm{Cl})\end{array}$ & $\begin{array}{c}\boldsymbol{p} \\
\text { Paired } t \text {-tests }\end{array}$ & $\begin{array}{c}\boldsymbol{p} \\
\text { RM-ANOVA }\end{array}$ \\
\hline $\begin{array}{l}\text { PLASMA K2EDTA TUBES } \\
\quad \text { Immediate processing }\end{array}$ & $35.2 \pm 14.4$ & 100 & - & - \\
$\begin{array}{l}\text { SERUM TUBES } \\
\quad \text { Centrifuged after } 30 \mathrm{~min}\end{array}$ & $32.6 \pm 14.2$ & $91.8(0.89-0.95)$ & 0.59 & \\
$\quad$ Centrifuged after $60 \mathrm{~min}$ & $32.7 \pm 14.6$ & $91.9(0.89-0.95)$ & 0.62 & 0.84 \\
$\quad$ Centrifuged after $120 \mathrm{~min}$ & $32.4 \pm 14.8$ & $91.1(0.88-0.94)$ & 0.59 & \\
\hline
\end{tabular}

Table 1. Retrieval in percent of the absolute PTH values. Plasma samples in K2EDTA tubes were compared to serum tubes using paired t-tests. To look for a possible time trend following delayed processing of serum samples a repeated measures analysis of variance (RM-ANOVA) was used.

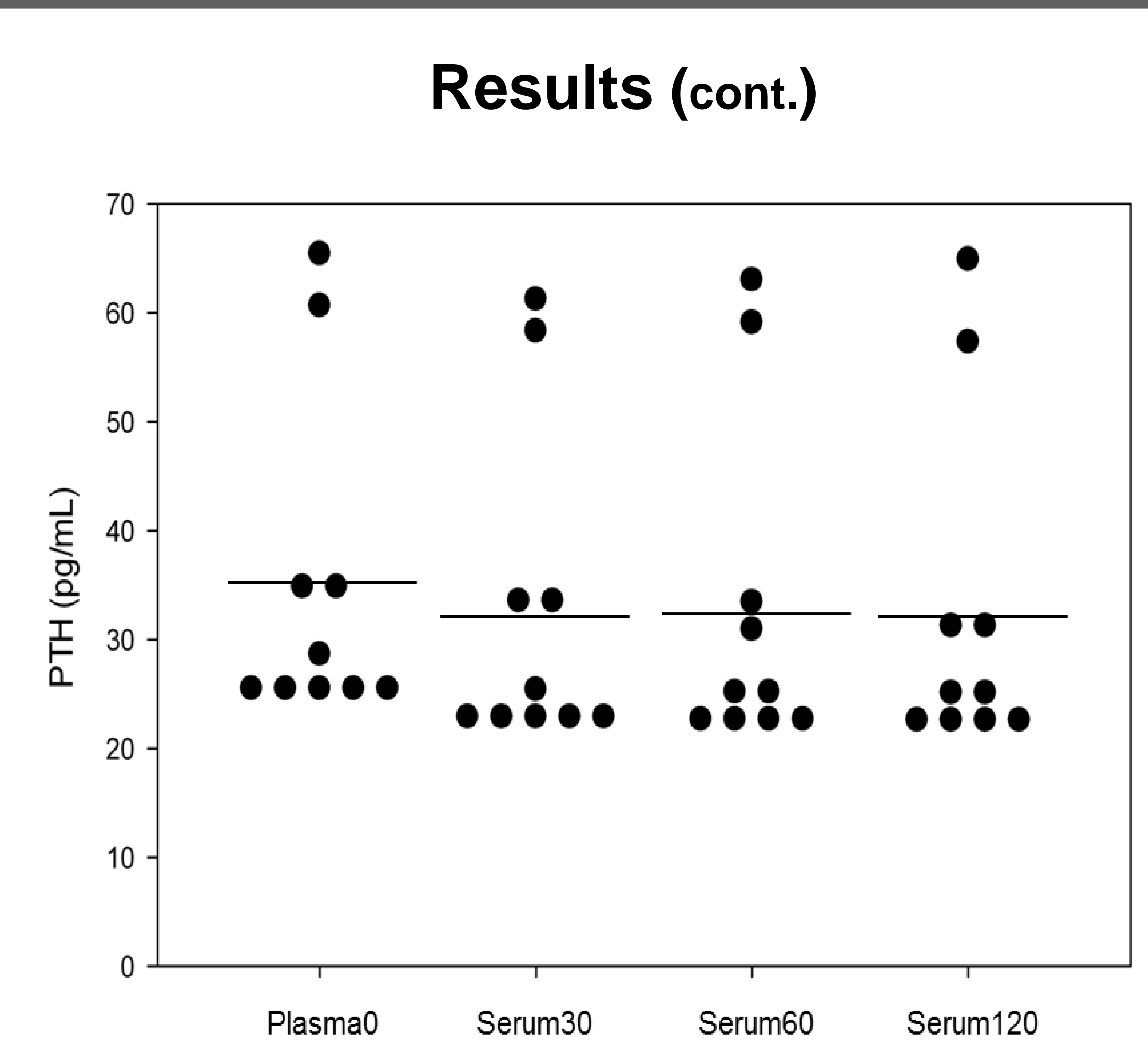

Fig. 1. Effect of sample type and processing on $\mathrm{PTH}$ values with Elecsys $\mathrm{PTH}$ immunoassay. Plasma0, PTH values in EDTA plasma (gold standard). Serum30, Serum60 and Serum120, PTH values in serum processed 30,60 and 120 minutes after sample collection.

In serum tubes stored for five years, PTH values were significantly elevated compared to the reference interval as tested by a binomial test and a one sample t-test $(p<0.01)$.

No significant difference between PTH from the five-year old serum samples and matched K2EDTA tubes measured from March to April 2007 was found using a paired t-test $(p=0.58)$. The matched samples used for comparison were analyzed using a sandwich ELISA (cat. No 7022, Biomerica, Newport Beach, CA, USA), and all values were corrected by the formula $(X+12) / 1.70$ in order to achieve identity with data using Elecsys Intact PTH (Roche Diagnostics, Mannheim, Germany).

\section{Conclusion}

This is to our knowledge the first study to investigate the stability of PTH in samples stored for five years.

- The study demonstrates that it is possible to obtain valid PTH results in serum samples stored at $-20^{\circ} \mathrm{C}$ for five years.

- The PTH retrieval in serum compared to plasma was acceptable, and delayed processing for up to 120 minutes did not affect PTH stability.

- To match results obtained in serum with previously reported data measured in K2EDTA plasma samples, adjustment by a retrieval factor of 0.92 should be considered.

\section{References \& Acknowledgements}

(1) Cavalier E, Carlisi A, Bekaert AC, Rousselle O, Chapelle JP, Delanaye P. New insights on the stability of the parathyroid hormone as assayed by an automated 3rd generation pth assay. Clinica chimica acta; international journal of clinical chemistry 2012;413:353-4.

(2) Cavalier E, Delanaye P, Carlisi A, Krzesinski JM, Chapelle JP. Stability of intact parathyroid hormone in samples from hemodialysis patients. Kidney international 2007;72:370-2. in serum and edta plasma from hemodialysis patients. Clinical chemistry 2004;50:1713-4.

(4) Scharnhorst V, Valkenburg J, Vosters C, Vader H. Influence of preanalytical factors on the immulite intact parathyroid hormone assay. Clinical chemistry 2004;50:974-5.

(5) Holmes DT, Levin A, Forer B, Rosenberg F. Preanalytical influences on dpc immulite 2000 intact pth assays of plasma and serum from dialysis patients. Clinical chemistry 2005;51:915-7.

Conflict of Interest Camilla S. Andersen: affiliated to Aalborg University in Denmark to conduct clinical research and employed by Center for Clinical \& Basic Research (CCBR). Peter Vestergaard and Parisa Gazerani: affiliated to Aalborg University in Denmark to conduct clinical research for the Faculty of Medicine. Hans C. Hoeck: employed by CCBR, a private re-search company engaged in contract research with various pharmaceutical and biotech companies.

We are indebted to the clinical staff at the CCBR laboratory department in Aalborg and to the Synarc $\Theta$ Research Laboratory for their partnership and participation in this project. 